\title{
Néphrologie et neurobiologie : un canal qui fait le pont...
}

Ice canal sodium épithélial est le facteur limitant de la réabsorption sodique dans les parties distales du néphron, du colon et, de façon générale, dans tous les tissus épithéliaux qui répondent aux hormones minéralo-corticoïdes, telle que l'aldostérone. Ce canal joue donc un rôle important dans l'homéostasie du sodium extracellulaire et dans le contrôle du volume sanguin et de la pression sanguine; il semble également jouer un rôle notable dans le contrôle de la réabsorption des sécrétions pulmonaires. Finalement, c'est le récepteur d'une classe de médicaments diurétiques, tels l'amiloride et le triamtérène.

L.'identification du canal sodium épithélial s'est révélée particulièrement délicate, car peu de cellules l'expriment, et seulement en très petites quantités. Les approches biochimiques n'ont pas permis l'identification des molécules composant la structure du canal proprement dit. (irâce à des techniques de clonage par expression fonctionnelle dans l'ovocyte de la grenouille Xenopus laevis, il a été possible, l'an passé, d'identifier une sous-unité de ce canal dans notre laboratoire [1] et dans celui de M. Lazdunski à Nice [2].

Nous avons appelé cette sous-unité a rENaC pour "sous-unité a du canal épithélial sodique du rat " (rat epithelial $\mathrm{Na}$ channel). Bien que l'expression de cette sous-unité dans l'ovocyte permette la reconstitution d'un canal épithélial sensible à l'amiloride, il n'était pas possible, dans ce système, de reconstituer la totalité de l'activité caractérisée dans les tissus épithéliaux. Il était ainsi d'emblée apparent que certains composants du canal manquaient. Grâce à un essai de complémentation fonctionnelle, il a été possible d'identifier deux nouvelles sous- unités ( $\beta$ et $\gamma$ rENaC) à partir de cellules épithéliales coliques du rat. Ia coexpression des trois sous-unités dans l'ovocyte permet alors la reconstitution d'un canal qui a toutes les propriétés physiologiques et pharmacologiques du canal natif [3]. La stoechiométrie des sous-unités du canal sodium n'a pas encore été déterminée. A l'image de ce que l'on sait pour d'autres canaux cationiques déjà identifiés, une structure tétramérique (comme cela est proposé pour le canal potassium sensible au voltage) ou une structure pentamérique (comme proposé pour le récepteur de l'acétylcholine de type nicotinique) sont possibles. La structure hétero-multimérique de ce canal semble de toute façon particulière, chaque sous-unité n'étant insérée dans la membrane que par deux segments hydrophobes et exposant un large domaine protéique à l'extérieur de la cellule.

Un des éléments les plus intéressants de cette découverte est l'existence d'une grande similitude de structure entre les sous-unités du canal épithélial sodium et une famille de protéines identifiées il y a trois ans par le groupe du Dr Martin Chalfie à Columbia (New York, USA), et qu'il a appelées "dégénérines". I es gènes codant pour ces protéines ont été identifiés chez un invertébré, le ver nématode Caenorhabditis elegans, un modèle très populaire à l'heure actuelle pour l'étude du développement du système nerveux (voir ce numéro de $\mathrm{m} / \mathrm{s}$, p. 337). Dans le cas particulier, le groupe de Chalfie voulait identifier des gènes impliqués dans la différenciation des neurones sensibles au toucher et à la pression (neurones mécanosensibles). Le groupe de Chalfie a ainsi identifié une série de gènes (mec-4, mec-10, deg-1) qui, quand ils sont mutés, confêrent l'insensibilité de l'animal au toucher. De plus, certains mutants dominants ont un phénotype étomnant: le neurone sensitif se différencie normalement, puis, soudain, gonfle et, finalement, est lysé, comme s'il souffrait d'un trouble de perméabilité membranaire important. Il est donc probable que le groupe des dégénérines de l'invertébré appartient à une famille de protéines constituant un canal cationique (sodique ou calcique, par exemple) qui serait spécifiquement impliqué dans la transduction mécanique. Cette découverte est importante car, à l'heure actuelle, si l'on commaît beaucoup de transmetteurs impliqués dans les voies efférentes (motrices, par exemple), on ne connaît encore pratiquement rien des transmetteurs impliqués dans les voies afférentes (toucher, audition, goût, douleur, proprioception etc.). Il semblerait donc que l'on soit en train de découvrir une toute nouvelle famille de gènes qui codent pour des canaux cationiques et qui pourraient être exprimés dans un très grand nombre de cellules et de tissus. On peut même faire l'hypothèse que le gène ancestral est extrêmement ancien; cela sur la base de la constatation que les sous-unités du canal sodium, bien que dérivant du même gène ancestral, ne partagent que 30 à $35 \%$ d'identité au niveau de leur structure protéique; la duplication du gène ancestral doit donc être extrêmement ancienne. A l'heure actuelle, nous postulons que le gène ancestral coderait pour un canal cationique que l'on trouverait dans les cellules procaryotes (bactéries, algues) et dans les cellules eucaryotes unicellulaires (levures) impliqué dans le contrôle du volume cellulaire. Cette famille de canaux aurait évolué chez les invertébrés et vertébrés, non seulement pour contrôler 
le volume cellulaire et pour assurer des fonctions spéciales, en particulier dans le système nerveux central et périphérique (sensibilité profonde, toucher, éventuellement douleur, perception des sons, perception des goûts, etc.), mais aussi chez les vertébrés supérieurs pour le contrôle de l'homéostasie du volume extracellulaire (canal épithélial sensible à l'amiloride).

Quelles sont les implications cliniques éventuelles de cette découverte? Premièrement, le canal épithélial sodium sensible à l'amiloride pourrait être impliqué dans certaines formes rares d'hypertension familiale, en particulier dans le syndrome de Liddle*. Il sera également intéressant de voir dans quelle mesure le canal lui-même, ou sa régulation, sont perturbés dans certaines formes d'hypertension artérielle essentielle. Un deuxième intérêt est l'étude de l'activité du canal sodium sensible à l'amiloride durant le développement et la maturation pulmonaire. On sait que, chez le nouveau-né prématuré, le poumon n'est pas capable de réabsorber efficacement les sécrétions alvéolaires et bronchiques. (cela pourrait être en partie dû à l'immaturité du canal sodique épithélial $[6,7])$. On pense également que le canal épithélial sodium sensible à l'amiloride pourrait jouer un rôle dans l'expression du gène de la mucoviscidose (CFTR). En effet, les patients souffrant de mucoviscidose peuvent être améliorés par l'inhalation pulmonaire d'amiloride, suggérant qu'un flux sodique de réabsorption pathologique contribue à la viscosité des sécrétions bronchiques. Il est tentant, enfin, de faire l'hypothèse que l'expression pathologique de certains types de ces canaux cationiques dans le système nerveux central pourrait, dans certaines circonstances cliniques, conduire à des maladies neuro-dégénératives.

On accuse souvent la biologie moléculaire d'être par trop réductionniste, voire simpliste. C'est souvent

\footnotetext{
* Syndrome héréditaire caractérisé par une hypertension artérielle, une hypokaliémie, une alcalose et une aldostéronurie basse. Is mécanisme de l'ano malie tubulaire rénale responsable de ce syndrome
}

vrai. Il n'en reste pas moins que l'identification de gènes importants et conservés durant toute l'évolution permet, au contraire, de créer soudain des ponts entre des domaines biologiques et des disciplines cliniques qui semblaient, à première vue, bien éloignés les uns des autres. Ainsi néphrologie, pneumologie et neurobiologie voient converger certains de leurs intérêts grâce, non pas à un pont, mais à un canal!

B.R.
1. Canessa CM, Horisberger JD, Rossier BC. E.pithelial sodium channel related to proteins involved in neurodegeneration. Nature 1993; $61: 467-470$.

2. I.ingueglia E, Voilley N, Waldmann R, I adzunski M, Barbry P. Expression cloning of an epithelial amiloride-sensitive $\mathrm{Na}^{+}$channel. A new channel type with homologies to Cae norabditis elegans degenerins. FEBS Lett 1993 ; 318: 95-9.

3. Canessa CM, Schild L, Buell G, Thorens B, Gautschi I, Horisberger JD, Rossier B. Amiloride-sensitive epithelial $\mathrm{Na}^{+}$channel is made of three homologous subunits. Nature 367, 463-467 (1994).

4. Horisberger JD, Canessa CM, Rossier BC. The epithelial $\mathrm{Na}^{+}$channel recent developments. (ell Phy iol Biochem 1993; 3 : 283-94.

5. Driscoll M. Molecular genetics of cell death in the nematode Caenorabditis elegans. J. Neurobiol 1992; 23: 1327-51.

6. O’Brodovich H. Canessa CM, Ueda J Rafil B, Rossier BC, Edelson J. Expression of the epithelial $\mathrm{Na}^{+}$channel in the developing rat lung. Am I Physiol 1993; 265 (Cell Physiol 34) : C491-6.

7. Voilley N, Lingueglia E, Champigny G, Mattei MG, W'aldmann $R$, Lazdunski $M$, Barbry P. The lung amiloride-sensitive $\mathrm{Na}^{+}$ channel: biophysical properties, pharmacology, ontogenesis, and molecular cloning. Proc Nall Acad Sci USA 1994 ; 91 : 247-251. 